\title{
First description of food-borne Salmonella enterica resistance regions R1 and R3 associated with IS26 elements
}

\author{
Eduarda Gomes-Neves $^{\mathrm{a}, \mathrm{b}, 1}$, Vera Manageiro ${ }^{\mathrm{b}, \mathrm{c}, 1}$, Eugénia Ferreira ${ }^{\mathrm{c}}$, José M. Correia da Costa ${ }^{\mathrm{b}, \mathrm{c}}$, \\ Manuela Caniça ${ }^{\mathrm{c}, *}$
}

a ICBAS, Instituto de Ciências Biomédicas Abel Salazar, Universidade do Porto, Porto, Portugal

${ }^{\mathrm{b}}$ CECA-ICETA, Centro de Estudos de Ciência Animal, Universidade do Porto, Porto, Portugal

${ }^{\mathrm{c}}$ Departamento de Doenças Infeciosas, Instituto Nacional de Dr. Ricardo Jorge, Lisboa and Porto, Portugal

Received 1 December 2014; accepted 13 March 2015

Available online

\begin{abstract}
In this study, we assessed the presence of IS26 in food-borne ASSuT-type Salmonella enterica isolates. A new genetic region (R3) was described, that included a C14 caspase gene between IS26 elements. R3 was present in two Salmonella Rissen isolates from a swine carcass and a meat handler, collected at the same abattoir. Furthermore, a new rearrangement of resistance region R1, harboring the bla $a_{\mathrm{TEM}-1}$ gene flanked by IS26 elements, was identified in Salmonella Typhimurium and Salmonella 4,[5],12:i:-, from different samples. This study highlights the zoonotic potential of Salmonella spp. isolates and the possible role of IS26 in the mobilization of resistance genes.
\end{abstract}

(C) 2015 Institut Pasteur. Published by Elsevier Masson SAS. All rights reserved.

\section{Introduction}

Salmonella enterica, like Salmonella Typhimurium DT104, the monophasic variant Salmonella 4,[5],12:i:- and Salmonella Rissen, has been involved in important multidrug-resistant (MDR) human infections associated with several sources [1-3]. The 4,[5],12:i:- variant has been linked to poultry, cattle, pig and pork products, with pigs being the likely reservoir of infection [1]. Indeed, as recently demonstrated, animal-husbandry-associated environments seem to contribute to enhancing Salmonella's pathogenic potential [2]. In fact, in

\footnotetext{
* Corresponding author.

E-mail addresses: egomesneves@mail.icav.up.pt (E. Gomes-Neves), vera. manageiro@insa.min-saude.pt (V. Manageiro), eugenia.ferreira@insa.minsaude.pt (E. Ferreira), jose.costa@insa.min-saude.pt (J.M. Correia da Costa), manuela.canica@insa.min-saude.pt (M. Caniça).

${ }^{1}$ These authors contributed equally to the work.
}

those isolates, resistance genes are often associated with integrons, insertion sequences (IS) and transposons, clustering within chromosomal antimicrobial resistance regions [4]. IS26 has been particularly implicated in the dissemination of chromosomal regions containing resistance genes by facilitating their mobilization between distinct genetic areas [5].

Indeed, a $d f r A 5$-IS26 configuration was previously detected among Escherichia coli strains with different serotypes sourced from both humans and animals, acting as a conduit for the transfer of integron-related resistance genes to human pathogens [6]. In Salmonella strains, namely $S$. Typhimurium and the monophasic variant $S .4$ [5],12:i:-, IS26 elements have been linked to the presence of resistance regions conferring an R-type ASSuT resistance pattern [7,8].

In this study, we investigated the genetic environment of bla $_{\text {TEM-1 }}$ genes among multidrug-resistant $S$. enterica isolates, in order to elucidate the genetic relationship of IS26 mobile genetic elements towards these important resistance genes. 


\section{Materials and methods}

\subsection{Sampling and phenotype characterization}

A collection of 60 MDR (R-type ASSuT phenotype) serotyped $S$. enterica strains (32 S. Typhimurium and 3 monophasic variants $S$. 4,[5],12:i:-, $11 S$. Derby, $4 S$. Rissen, 3 $S$. London, $3 S$. Mbandaka, $2 S$. Give, $1 S$. Enteritidis and $1 S$. Sandiego), isolated from slaughtered swine samples, were recovered from ileocecal lymph node samples of swine, carcass swabs, meat samples and meat handlers' hands, in a previous study $[9,10]$.

\subsection{Analysis of sequences flanking IS26 elements}

In all 60 MDR $S$. enterica isolates, we investigated the genetic organization of three resistance regions (named R1, R2 and R3), and the association of IS26 with resistanceencoding genes, by PCR mapping and sequencing assays,

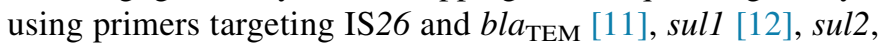
sul3, merD and urf2 (Table 1). Controls were included in all assays: Salmonella spp. strains [10] were used as PCRpositive controls for bla $a_{\mathrm{TEM}}$, sull, sul2 and sul3; E. coli INSRA1169 [13] for merD and urf2; and E. coli INSLA51 [12] for the IS26 gene, respectively. Sequence alignments and generation of resistance cassette contigs were performed using Bionumerics software (Applied Maths). Gene identity was confirmed at the NCBI website (http://www.ncbi.nlm.nih. gov/).

\subsection{Statistical analysis}

OpenEpi software, version 3 (www.openepi.com) was used for statistical analysis. Two-sided $P$ values of $<0.05$ were considered to be statistically significant.

\section{Results and discussion}

PCR mapping identified IS26 elements in 23/60 (38.3\%) of MDR S. enterica isolates in three different resistance regions (R1, $\mathrm{R} 2$ and R3), statistically associated with antibiotic (bla $a_{\mathrm{TEM}-1}$, region R1 and R3) or mercury (merD-merE-urf2, region R2) resistance-encoding genes (Table 2), with $P<0.001$. Overall, we detected 27 bla $_{\mathrm{TEM}-1}$ genes, from which 21 were flanked up- and downstream by two copies of IS26, in a Tn6029-like structure that

Table 1

Primers designed in this study and used for PCR mapping analysis.

\begin{tabular}{lll}
\hline Gene & Primer name & Primer sequence $\left(5^{\prime} \rightarrow 3^{\prime}\right)$ \\
\hline sul2 & Sul2-F & ATGAATAAATCGCTCATCATTTTC \\
& Sul2-R & TTAACGAATTCTTGCGGTTTC \\
sul3 & Sul3-F & ATGAGCAAGATTTTTGGAATC \\
& Sul3-R & CTAACCTAGGGCTTTGGA \\
merD & MerD-F & CCTTCGAGGCGGGTATC \\
urf2 & Urf2-R & TGTTGCAGGCAGGAATAGC \\
\hline
\end{tabular}

was described by Cain et al. [14]: the R1 region (17 S. Typhimurium and $2 S$. 4,[5],12:i:-) and, here firstly described, the R3 region (2 $S$. Rissen) (Fig. 1A, Fig. 1B).

As shown in Fig. 1A, in $S$. Typhimurium strains (including monophasic strains), the genetic arrangement (3540 bp) comprised between the two IS26 was identical to the chromosomal resistance R1 region (GenBank accession no. HQ331538) previously described in epidemiologically unrelated Salmonella strains recovered only from humans in Italy [7]. However, sul1, sul2 and sul3 resistance genes were not present in the R1 surrounding regions in this study, revealing a different genetic arrangement in our animal and meat handler isolates when compared to human Salmonella cases in Italy and Canada [7,15].

In two $S$. Rissen isolates (Fig. 1B) recovered in the same abattoir, from a carcass and a meat handler, the new resistance region (R3) presented an IS26-bla TEM-1 $_{\text {genetic platform (with }}$ $1857 \mathrm{bp}$ ); this structure was followed by a peptidase C14 caspase catalytic subunit P20-encoding gene, plus another copy of IS26 found downstream of the $b l a_{\mathrm{TEM}-1}$ gene (with $1455 \mathrm{bp}$ ). Interestingly, the C14 caspase gene was also found in an Actinomycete integrating conjugative element, which catalyzed the mobilization of other genetic elements such as genomic islands and virulence plasmids [16]. The truncated $\mathrm{Tn} 3$ transposon of the $\mathrm{R} 1$ region was not present in this R3 genetic region (Fig. $1 \mathrm{~B}$ ).

The subsequent use of primers specific to known resistance-encoding genes generated a PCR amplicon (R2 region, $3447 \mathrm{bp}$ ) both in the $21 \mathrm{~S}$. Typhimurium (including the three monophasic variants) and the $2 \mathrm{~S}$. Rissen isolates (Table 2 and Fig. 1C). Indeed, this region was the only one identical to that described by Lucarelli et al. (GenBank accession no. HQ331538), presenting part of a mercury resistance operon and flanked downstream by an IS26 element [7]. This operon (which has been reported to be a conserved region among Salmonella strains) is of great concern, since its co-existence with antibiotic resistance regions suggests indirect selection of antibiotic resistance in $S$. enterica strains [7,17].

Insertion sequence IS26 plays a key role in dissemination of antibiotic resistance genes, namely in Salmonella spp. $[5,6,18]$, both in plasmids and in chromosomal genomic islands $[19,20]$. In this study, we described three different chromosomal regions containing antibiotic or mercury resistance genes that are flanked by, and eventually interspersed with, copies of IS26, including the new R3. The presence of multiple copies of IS26 enhances the mobilization of large MDR regions, which might include resistance and pathogenicity-encoding genes and then build new MDR regions. Indeed, a recent study indicated that transposition of IS26, presumably donated by plasmids originally acquired by biphasic $S$. Typhimurium, was involved in the deletion of the $f j A B$ operon and surrounding genes and hence was responsible for the monophasic phenotype of $S$. 4,5,12:i:isolates [21].

In conclusion, the presence of RR1 plus RR2 in both $S$. Typhimurium and in its monophasic variant (previously 
Table 2

Characteristics of 28 Salmonella isolates containing resistance regions (R1, R2, R3) and/or producing TEM-1 $\beta$-lactamase.

\begin{tabular}{|c|c|c|c|c|c|c|}
\hline \multirow{2}{*}{$\begin{array}{l}\text { Serotype (no. } \\
\text { of isolates) }\end{array}$} & \multirow{2}{*}{$\begin{array}{l}\text { Resistance } \\
\text { phenotype }^{\text {a }}\end{array}$} & \multirow{2}{*}{$\begin{array}{l}\text { Sampled material } \\
\text { (number of isolates) }\end{array}$} & \multirow{2}{*}{$\begin{array}{l}b l a_{\text {TEM-1 }} \\
(\mathrm{n}=27)\end{array}$} & \multicolumn{3}{|l|}{ Resistance region $^{\mathrm{b}, \mathrm{c}}$} \\
\hline & & & & $\begin{array}{l}\mathrm{R} 1 \text { (IS26-tnp3R } \Delta \\
- \text { bla }_{\mathrm{TEM}-1 \mathrm{~b}} \text {-tnpB-IS26) } \\
(\mathrm{n}=19)\end{array}$ & $\begin{array}{l}\mathrm{R} 2 \\
\text { (merD-merE-urf2-tniA } \\
\Delta 1 \text {-IS26) }(\mathrm{n}=23)\end{array}$ & $\begin{array}{l}\mathrm{R} 3 \\
\text { (IS26-bla } \\
\text { 14-IS26) }(\mathrm{n}=2)\end{array}$ \\
\hline \multirow[t]{2}{*}{ 4,[5],12:i:- (3) } & ASSuT & Meat (1); lymph node (1) & + & + & + & - \\
\hline & ST & Lymph node (1) & - & - & + & - \\
\hline \multirow[t]{6}{*}{ Typhimurium (20) } & ASSuT & $\begin{array}{l}\text { Lymph node (5); } \\
\text { carcass (5); Meat (5) }\end{array}$ & + & + & + & - \\
\hline & AST & Meat handler (1) & + & + & + & - \\
\hline & AT & Lymph node (1) & + & + & + & - \\
\hline & & Carcass (1) & + & - & + & - \\
\hline & ASuTW & Lymph node (1) & + & - & - & - \\
\hline & CASSuT & Meat (1) & + & - & - & - \\
\hline \multirow[t]{3}{*}{ Rissen (3) } & CASSuTW & Carcass (1) & + & - & + & + \\
\hline & ASSuTW & Meat handler (1) & + & - & + & + \\
\hline & ASSuT & Lymph node (1) & + & - & - & - \\
\hline London (2) & ANSSuT & Carcass (2) & + & - & - & - \\
\hline
\end{tabular}

a A, ampicillin; C, chloramphenicol; S, streptomycin; Su, sulfamethoxazole; T, tetracycline; W, trimethoprim.

b + , positive result; - , negative result.

c Regions presented in Fig. 1.

reported only in human isolates), as well as RR2 plus RR3 in $S$. Rissen from diverse food-chain-related reservoirs, highlights the zoonotic potential of such isolates and the possible role of IS26 in the mobilization of resistance genes within, to and from animal settings. Thus, it is imperative and mandatory to view food-producing animals as reservoirs of non-typhoidal Salmonella in order to monitor this situation in humans, animals and food.

A RR1 (3540 bp): S. Typhimurium ( $n=17)$ and variant 4,[5],12:i:- $(n=2)$

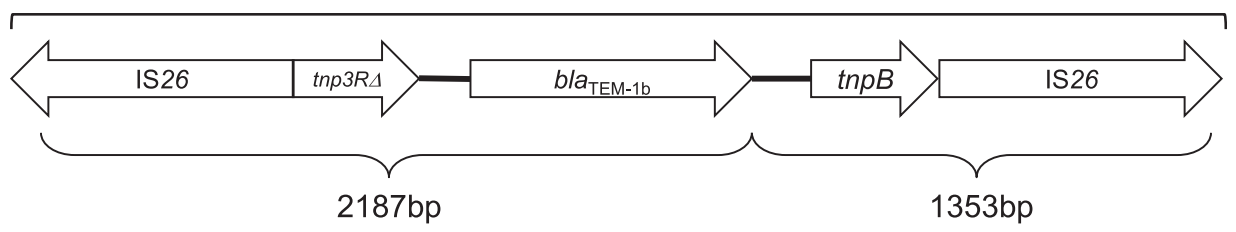

B New RR (RR3) (>3312 bp): S. Rissen ( $n=2)$

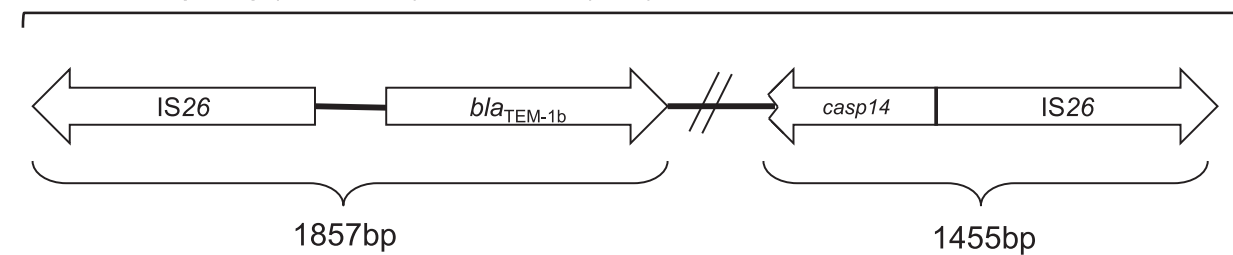

C $\quad$ RR2 (3447 bp): S. Typhimurium $(n=18)$, variant 4,[5],12:i:- $(n=3)$, and S. Rissen $(n=2)$

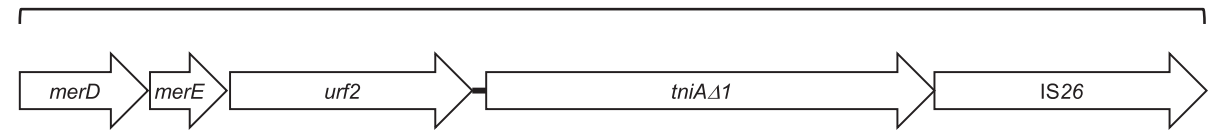

Fig. 1. Schematic representation of the genetic environment of resistance genes in R-type ASSuT S. enterica isolates. A: sequence of resistance region R1 (3540 bp) of $17 \mathrm{~S}$. Typhimurium isolates and 2 monophasic variants harboring the bla $a_{\mathrm{TEM}-1}$ gene; B: the new R3 genetic organization of $b l a_{\mathrm{TEM}-1}$ (R3, $>3312 \mathrm{bp}$ ) found in $2 \mathrm{~S}$. Rissen isolates; C: genetic organization of mercury resistance operon (R2, $3447 \mathrm{bp}$ ), in both $18 \mathrm{~S}$. Typhimurium and 3 variants 4,[5],12:i:-, as well as 2 $S$. Rissen isolates. The directions of transcription of the corresponding genes are depicted by arrows. 


\section{Conflicts of interest}

There was no conflict of interest.

\section{Acknowledgments}

V. Manageiro was supported by grant SFRH/BPD/77486/ 2011 from the FCT, Lisbon, Portugal. This study was in part supported by NIH internal funding. The authors also thank the Fundação para a Ciência e a Tecnologia (FCT) for project grant PEst-OE/AGR/UI0211/2011-2014, Strategic Project UI211-2011-2014.

\section{References}

[1] Hopkins KL, Kirchner M, Guerra B, Granier SA, Lucarelli C, Porrero MC, et al. Multiresistant Salmonella enterica serovar 4,[5],12:i:in Europe: a new pandemic strain? Euro Surveill 2010;15:19580.

[2] Oliveira M, Wijnands L, Abadias M, Aarts H, Franz E. Pathogenic potential of Salmonella Typhimurium DT104 following sequential passage through soil, packaged fresh-cut lettuce and a model gastrointestinal tract. Int J Food Microbiol 2011;148:149-55.

[3] EFSA/ECDC. The European Union summary report on antimicrobial resistance in zoonotic and indicator bacteria from humans, animals and food in 2012. EFSA J 2014;2014(12):3590.

[4] Miriagou V, Carattoli A, Fanning S. Antimicrobial resistance islands: resistance gene clusters in Salmonella chromosome and plasmids. Microbes Infect 2006;8:1923-30.

[5] Harmer CJ, Moran RA, Hall RM. Movement of IS26-associated antibiotic resistance genes occurs via a translocatable unit that includes a single IS26 and preferentially inserts adjacent to another IS26. mBio 2014:5.

[6] Dawes FE, Kuzevski A, Bettelheim KA, Hornitzky MA, Djordjevic SP, Walker MJ. Distribution of class 1 integrons with IS26-mediated deletions in their 3'-conserved segments in Escherichia coli of human and animal origin. PLoS One 2010;5:e12754.

[7] Lucarelli C, Dionisi AM, Filetici E, Owczarek S, Luzzi I, Villa L. Nucleotide sequence of the chromosomal region conferring multidrug resistance (R-type ASSuT) in Salmonella Typhimurium and monophasic Salmonella Typhimurium strains. J Antimicrob Chemother 2012;67:111-4.

[8] Laorden L, Herrera-León S, Martínez I, Sanchez A, Kromidas L, Bikandi J, et al. Genetic evolution of the Spanish multidrug-resistant Salmonella enterica 4,5,12:i:- monophasic variant. J Clin Microbiol 2010;48:4563-6.

[9] Gomes-Neves E, Antunes P, Tavares A, Themudo P, Cardoso MF, Gärtner F, et al. Salmonella cross-contamination in swine abattoirs in
Portugal: carcasses, meat and meat handlers. Int J Food Microbiol 2012;157:82-7.

[10] Gomes-Neves E, Antunes P, Manageiro V, Gärtner F, Caniça M, da Costa JM, et al. Clinically relevant multidrug resistant Salmonella enterica in swine and meat handlers at the abattoir. Vet Microbiol 2014; 168:229-33.

[11] Mendonça N, Leitão J, Manageiro V, Ferreira E, Caniça M. Spread of extended-spectrum $\beta$-lactamase CTX-M-producing Escherichia coli clinical isolates in community and nosocomial environments in Portugal. Antimicrob Agents Chemother 2007;51:1946-55.

[12] Jones-Dias D, Manageiro V, Francisco AP, Martins AP, Domingues G, Louro D, et al. Assessing the molecular basis of transferable quinolone resistance in Escherichia coli and Salmonella spp. from food-producing animals and food products. Vet Microbiol 2013;167:523-31.

[13] Manageiro V, Ferreira E, Pinto M, Fonseca F, Ferreira M, Bonnet R, et al Two novel CMY-2-type $\beta$-lactamases encountered in clinical Escherichia coli isolates. Ann Clin Microbiol Antimicrob 2015;14:12.

[14] Cain AK, Liu X, Djordjevic SP, Hall RM. Transposons related to Tn1696 in IncHI2 plasmids in multiply antibiotic resistant Salmonella enterica serovar Typhimurium from Australian animals. Microb Drug Resist 2010;16:197-202.

[15] Mulvey MR, Finley R, Allen V, Ang L, Bekal S, El Bailey S, et al Emergence of multidrug-resistant Salmonella enterica serotype 4,[5],12:i:- involving human cases in Canada: results from the Canadian integrated Program on antimicrobial resistance surveillance (CIPARS), 2003-10. J Antimicrob Chemother 2013;68:1982-6.

[16] Ghinet MG, Bordeleau E, Beaudin J, Brzezinski R, Roy S, Burrus V. Uncovering the prevalence and diversity of integrating conjugative elements in actinobacteria. PLoS One 2011;6:e27846.

[17] Baker-Austin C, Wright MS, Stepanauskas R, McArthur JV. Co-selection of antibiotic and metal resistance. Trends Microbiol 2006;14:176-82.

[18] Djordjevic SP, Stokes HW, Chowdhury PR. Mobile elements, zoonotic pathogens and commensal bacteria: conduits for the delivery of resistance genes into humans, production animals and soil microbiota. Front Microbiol 2013;4:86.

[19] Doublet B, Praud K, Weill F-X, Cloeckaert A. Association of IS26composite transposons and complex In4-type integrons generates novel multidrug resistance loci in Salmonella genomic island 1. J Antimicrob Chemother 2009;63:282-9.

[20] Holt KE, Thomson NR, Wain J, Phan MD, Nair S, Hasan R, et al Multidrug-resistant Salmonella enterica serovar Paratyphi A harbors IncHIl plasmids similar to those found in serovar Typhi. J Bacteriol 2007; 189:4257-64.

[21] García P, Malorny B, Hauser E, Mendoza MC, Rodicio MR. Genetic types, gene repertoire, and evolution of isolates of the Salmonella enterica serovar 4,5,12:i:- spanish clone assigned to different phage types. J Clin Microbiol 2013;51:973-8. 